Supporting Information

\title{
Synthetic saponite clays as additives for reducing aging effects in PIM1 membranes
}

Federico Begni ${ }^{\mathrm{a}}$, Geo Paul ${ }^{\mathrm{a}}$, Elsa Lasseuguette ${ }^{\mathrm{b}}$, Enzo Mangano ${ }^{\mathrm{b}}$, Chiara Bisio ${ }^{\mathrm{a}, \mathrm{c}}$, MariaChiara Ferrari ${ }^{\mathrm{b}}$, Giorgio Gatti ${ }^{\mathrm{a}}$

aDipartimento di Scienze e Innovazione Tecnologica, Università degli Studi del Piemonte Orientale" Amedeo Avogadro", Viale Teresa Michel 11, 15121-Alessandria (Italy);

${ }^{b}$ School of Engineering, University of Edinburgh, Robert Stevenson Road, EH9 3FB, Edinburgh, UK

${ }^{c}$ CNR-SCITEC Istituto di Scienze e Tecnologie Chimiche “G. Natta”, Via C. Golgi 19, 20133-Milano (Italy).

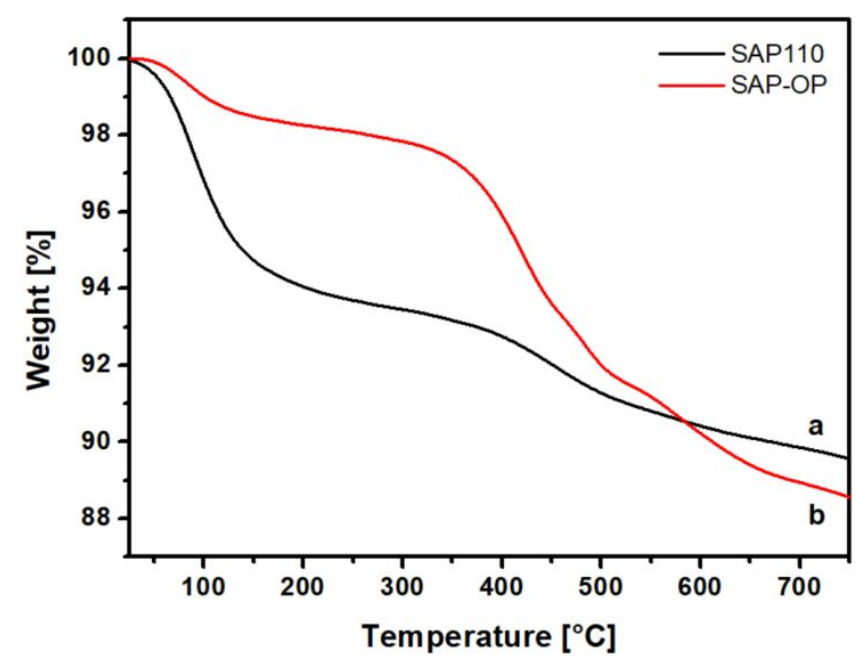

Figure S1. TGA curves of SAP110 (a) and SAP-OP (b) collected under Ar flow from 25 to $800{ }^{\circ} \mathrm{C}$. 


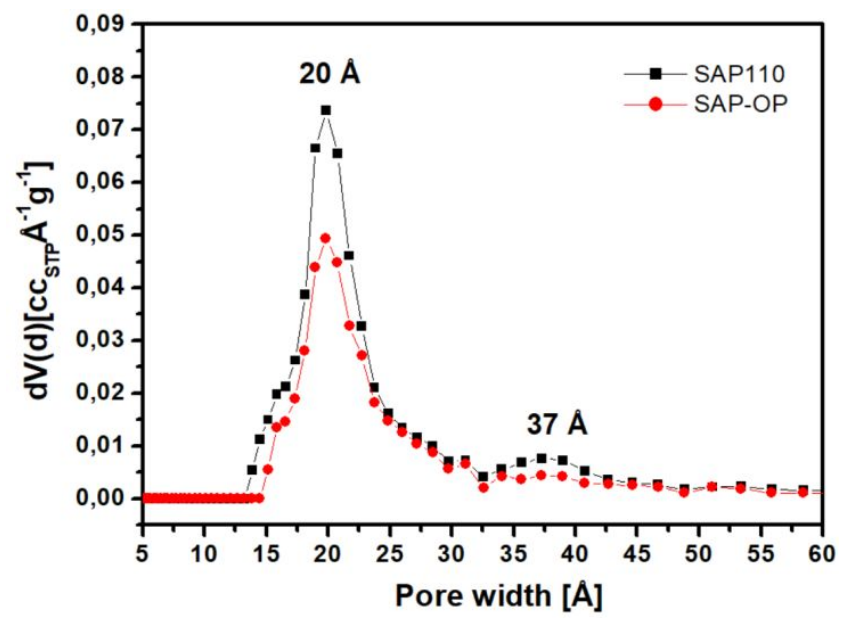

Figure S2. Pore size distribution of SAP110 ( $\square$ - $)$ and SAP-OP ( $\square-$

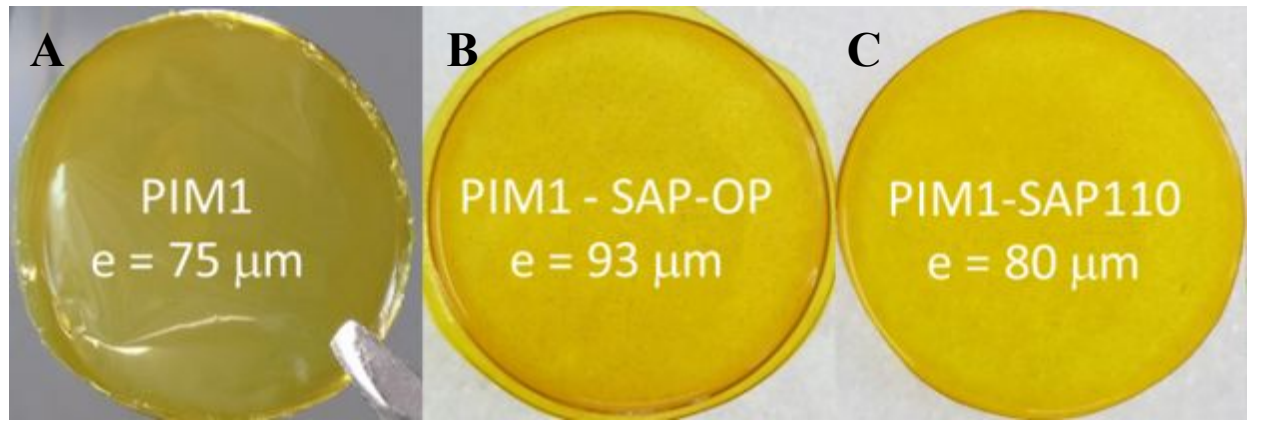

Figure S3. Pristine PIM1 (A) and MMMs (3\%wt) based on PIM1 plus SAP-OP (B) and SAP110 (C). (e = thickness in $\mu \mathrm{m})$. 


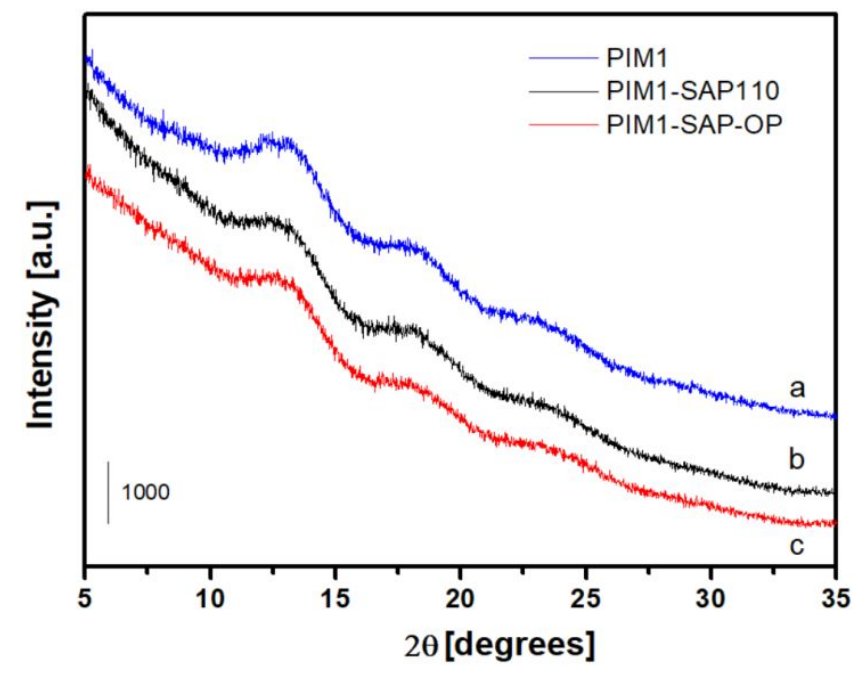

Figure S4. XRD pattern of pristine aged PIM1 membrane sample (a), aged PIM1-SAP110 (b) and aged PIM1SAP-OP (c).

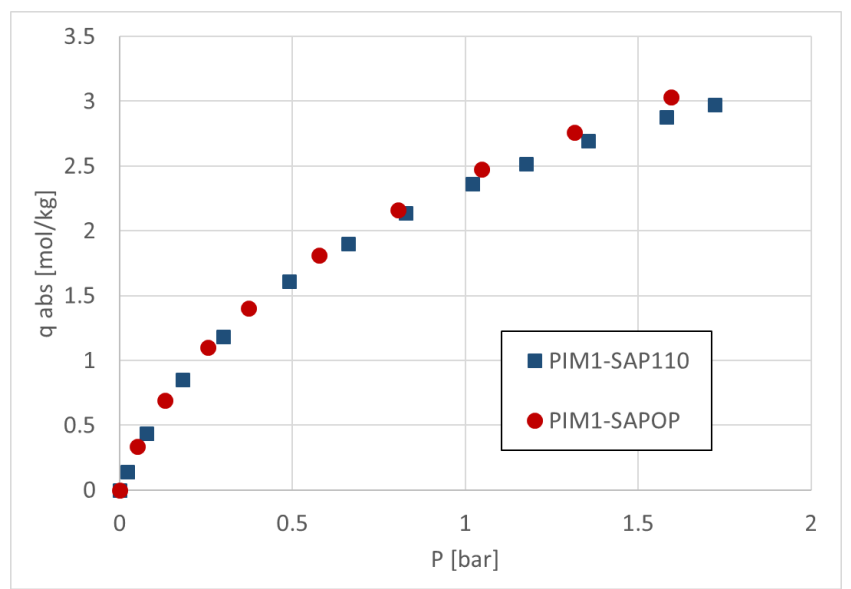

Figure S5. Carbon dioxide adsorption isotherms acquired at $273 \mathrm{~K}$ for mixed matrix samples.

Table S1. CHN analysis of SAP-OP sample

\begin{tabular}{|l|l|l|l|}
\hline Sample & $\% \mathrm{C}$ & $\% \mathrm{~N}$ & $\% \mathrm{CTA}^{+}$ \\
\hline SAP-OP & 3.62 & 0.35 & 6.75 \\
\hline
\end{tabular}


Table S2. ${ }^{13} \mathrm{C}$ Spin-lattice relaxation times $\left(\mathrm{T}_{1}\right)$ of various PIM1 based membranes.

\begin{tabular}{|c|c|c|c|c|c|c|c|c|c|}
\hline \multirow{3}{*}{$\begin{array}{c}{ }^{13} \mathrm{C} \\
\text { Chemical } \\
\text { shifts } \delta_{\mathrm{C}} \\
{[\mathrm{ppm}]}\end{array}$} & \multicolumn{9}{|c|}{${ }^{13} \mathrm{C}$ Spin-lattice relaxation times (s) } \\
\hline & \multicolumn{3}{|c|}{ PIM1 } & \multicolumn{3}{|c|}{ PIM1-SAP110 } & \multicolumn{3}{|c|}{ PIM1-SAP-OP } \\
\hline & $\begin{array}{l}\mathrm{MeOH} \\
\text { treated }\end{array}$ & $\begin{array}{c}\text { 3Months } \\
\text { aged }\end{array}$ & $\begin{array}{l}\text { 1Year } \\
\text { aged }\end{array}$ & $\begin{array}{c}\mathrm{MeOH} \\
\text { treated } \\
\text { fresh }\end{array}$ & $\begin{array}{l}\text { 3Months } \\
\text { aged }\end{array}$ & $\begin{array}{l}\text { 1Year } \\
\text { aged }\end{array}$ & $\begin{array}{c}\mathrm{MeOH} \\
\text { treated } \\
\text { fresh }\end{array}$ & $\begin{array}{l}\text { 3Months } \\
\text { aged }\end{array}$ & $\begin{array}{l}\text { 1Year } \\
\text { aged }\end{array}$ \\
\hline 148 & 9.37 & 8.82 & 9.25 & 8.92 & 10.98 & 9.16 & 9.43 & 9.64 & 8.32 \\
\hline 140 & 7.18 & 7.14 & 7.44 & 7.40 & 9.52 & 6.86 & 7.32 & 7.71 & 6.85 \\
\hline 110 & 5.89 & 5.64 & 5.87 & 5.59 & 7.39 & 5.95 & 6.10 & 6.18 & 5.78 \\
\hline 94 & 6.83 & 7.44 & 8.40 & 7.07 & 9.27 & 6.26 & 7.04 & 8.17 & 7.06 \\
\hline 58 & 8.44 & 8.85 & 9.29 & 8.46 & 10.12 & 9.58 & 7.32 & 8.81 & 8.60 \\
\hline 43 & 5.59 & 5.58 & 5.00 & 5.14 & 6.18 & 5.90 & 5.13 & 5.50 & 5.31 \\
\hline 30 & 0.319 & 0.370 & 0.346 & 0.386 & 0.374 & 0.341 & 0.358 & 0.367 & 0.402 \\
\hline
\end{tabular}

